\title{
Effect of Relative Permeability on Control Performance of Suspension System
}

\author{
Zhen $\mathrm{Jia}^{1, \mathrm{a}}$, Jie $\mathrm{Li}^{2, \mathrm{~b}}$ and Guan Chun $\mathrm{Li}^{2, \mathrm{c}}$ \\ ${ }^{1}$ School of Engineering Research Center of Maglev Technology, National University of Defense \\ Technology, Changsha 410000, China; \\ ${ }^{2}$ School of Engineering Research Center of Maglev Technology, National University of Defense \\ Technology, Changsha 410000, China; \\ a1031917012@qq.com, ${ }^{\mathrm{a} j i e l i @ n u d t . e d u . c n, ~}{ }^{\mathrm{C}}$ liguanchun_9@aliyun.com
}

Keywords: relative permeability, suspension control.

\begin{abstract}
In order to study the influence of relative permeability of track material in the performance of EMS suspension control, the Single Magnetic Model is established. Then we acquire good suspension performance by designing proper inner and outer controllers. The effect of the relative permeability on suspension control is studied in both cases, light load and overload conditions, through Matlab simulation. The simulation results show that with the decrease of relative permeability, the dynamic performances of suspension control system become worse.
\end{abstract}

\section{Introduction}

In the course of modeling, the relative permeability of the material is often considered to be large, and the relative permeability has little influence on the performance of the system, thus it can be neglected directly [1,2]. However, this lacks the support from simulation and experimental results. In this paper, a model considering relative permeability is established, and an appropriate controller is designed to achieve good suspension control. The effect of relative permeability on suspension control performance is simulated by MATLAB.

\section{Physical Systems Modeling}

Single magnet - rail suspension system structure is shown in Figure1.Ignoring the winding leakage flux, which means $\phi_{L}=0$; ignoring the iron core and the rail magneto resistance, which means that the magnetic potential in the gap $\delta(t)$ is uniform (shown $\mathrm{z}(\mathrm{t})$ in the figure). Since the purpose of this paper is to study the influence of the relative permeability of the solenoid core on the performance of the suspension control, it must take into account the relative permeability factor. External interference is neglected in the process of modeling [3].

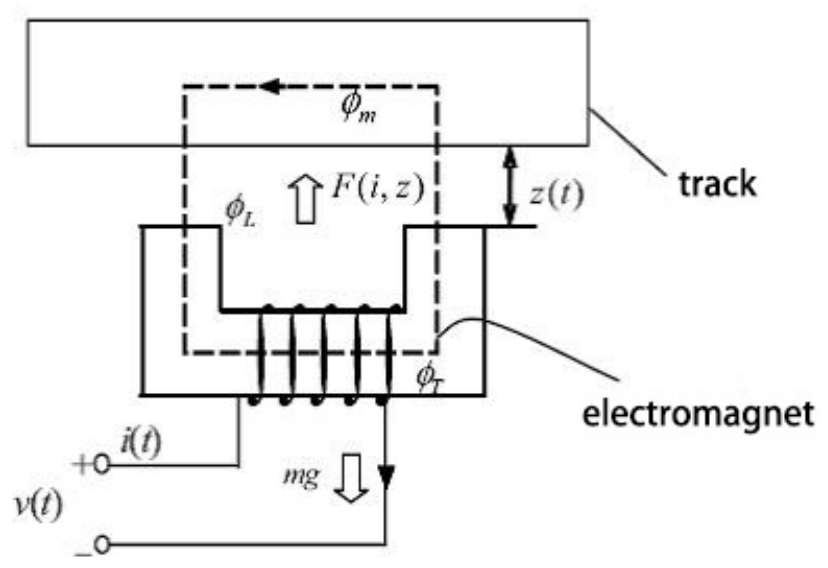

Fig 1 Single magnet - rail suspension system structure 
Table 1 The meaning of the letters in the model

\begin{tabular}{|c|c|c|c|}
\hline Letter & Meaning & Letter & Meaning \\
\hline$N$ & solenoid winding turns & $\mu_{0}$ & air permeability \\
\hline$\delta(t)$ & Air gap & $A$ & Core area \\
\hline$l$ & Length of magnetic circuit & $i(t)$ & Control coil current \\
\hline$B$ & Air - gap magnetic induction & $v(t)$ & the voltage of the control coil \\
\hline$\mu$ & Magnetic permeability & $\mu_{r}$ & Relative permeability \\
\hline
\end{tabular}

According to the Ampere Circle Theorem, we get:

$$
N I=\frac{B}{\mu_{0}} 2 \delta+\frac{B}{\mu_{0} \mu_{r}} l
$$

In which $l$ represents the length of the magnetic path other than the gap, so:

$$
B=\frac{\mu_{0} N i(t)}{\left(2 \delta(t)+\frac{l}{\mu_{r}}\right)}=\frac{\mu_{0} N i(t)}{2 \delta(t)+k}
$$

Column differential equations of system are written and linearized at the equilibrium point, then the state space equation of single-magnet model is established as follow: $(\Delta \delta, \Delta \dot{\delta}, \Delta i)$ is chosen as state variable.

$$
\left[\begin{array}{c}
\Delta \dot{\delta} \\
\Delta \ddot{\delta} \\
\Delta \dot{i}
\end{array}\right]=\left[\begin{array}{ccc}
0 & 1 & 0 \\
\frac{\mu_{0} N^{2} A i_{0}^{2}\left(2 \delta_{0}+k+4\right)}{\left(2 \delta_{0}+k\right)^{3} m} & 0 & -\frac{2 \mu_{0} N^{2} A i_{0}}{\left(2 \delta_{0}+k\right)^{2} m} \\
0 & \frac{2 i_{0}}{2 \delta_{0}+k} & -\frac{\left(2 \delta_{0}+k\right) R}{\mu_{0} N^{2} A}
\end{array}\right]\left[\begin{array}{c}
\Delta \delta \\
\Delta \dot{\delta} \\
\Delta i
\end{array}\right]+\left[\begin{array}{c}
0 \\
0 \\
\frac{\left(2 \delta_{0}+k\right)}{\mu_{0} N^{2} A}
\end{array}\right] \Delta v
$$

$$
\begin{gathered}
\Delta \delta=\left[\begin{array}{lll}
1 & 0 & 0
\end{array}\right]\left[\begin{array}{c}
\Delta \delta \\
\Delta \dot{\delta} \\
\Delta i
\end{array}\right]+\left[\begin{array}{l}
0 \\
0 \\
0
\end{array}\right] \Delta v \\
k_{1}=\frac{\mu_{0} N^{2} A i_{0}{ }^{2}\left(2 \delta_{0}+k+4\right)}{\left(2 \delta_{0}+k\right)^{3} m}, k_{2}=-\frac{2 \mu_{0} N^{2} A i_{0}}{\left(2 \delta_{0}+k\right)^{2} m}, k_{3}=\frac{2 i_{0}}{2 \delta_{0}+k}, k_{4}=-\frac{\left(2 \delta_{0}+k\right) R}{\mu_{0} N^{2} A}, k_{5}=\frac{\left(2 \delta_{0}+k\right)}{\mu_{0} N^{2} A} 。
\end{gathered}
$$

From the relationship between input and output and the state variables, control block diagram can be shown as Figure 2: 


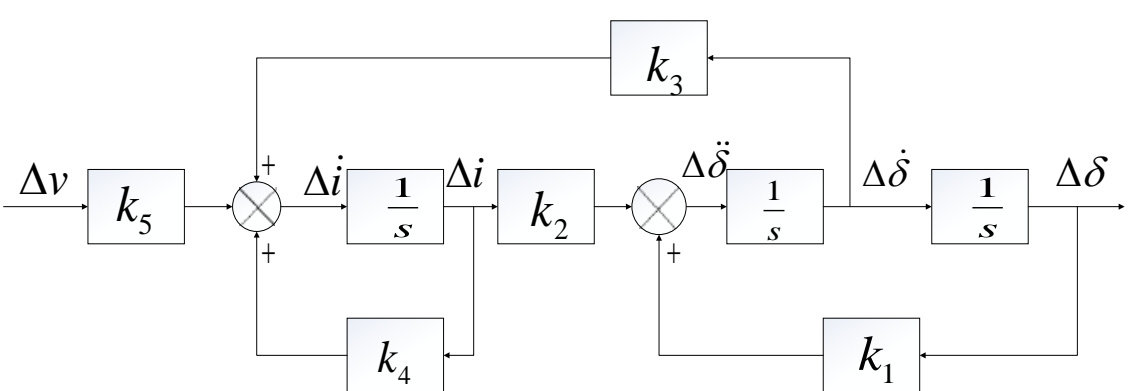

Fig 2 Single iron system model

\section{System Controller Design}

The model parameters are set according to the actual system measurement data :

The parameters for light load:

$$
N=360, \delta=0.009 \mathrm{~m}, A=0.028 \times 0.66 \mathrm{~m}^{2}, R=0.5 \Omega, \mu_{0}=4 \pi \times 10^{-7}, m_{0}=600 \mathrm{~kg},
$$
$i_{0}=23 A, l=0.3 m$ 。

The parameters for over load:

$$
N=360, \delta=0.009 \mathrm{~m}, A=0.028 \times 0.66 \mathrm{~m}^{2}, R=0.5 \Omega, \mu_{0}=4 \pi \times 10^{-7}, m_{0}=800 \mathrm{~kg},
$$
$i_{0}=35 A, l=0.3 m$ 。

System model parameters are different under light load and heavy load, therefore, the controller parameters are designed for them respectively. By comparing to the simulation results, we study the effects of the relative permeability on the system under both light and heavy load conditions.

The actual input of the system is the expected suspension clearance, $\Delta r=9 \mathrm{~mm}$; the actual output is the suspension clearance $\Delta \delta$. The proportional control is introduced into the inner loop. In the meantime, the closed-loop feedback and the PID controller are introduced into the outer loop for a good control [4].

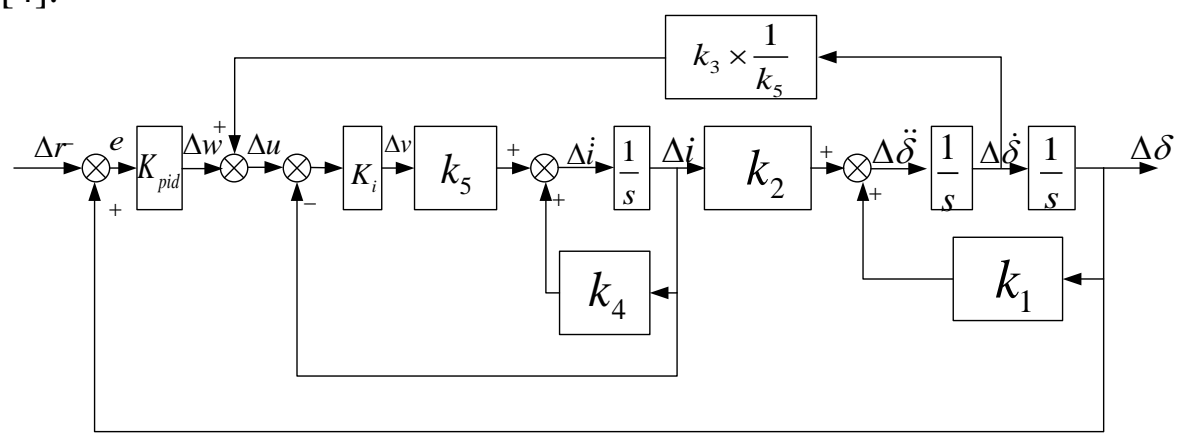

Fig 3 Control block diagram model

Parameter of proportional controller: $k_{i}=110$, and the parameters of PID controller are selected out by trial.

Parameters for light load:

$$
k_{P}=150000, k_{I}=100000, k_{D}=400
$$

Parameters for heavy load:

$$
k_{P}=190000, k_{I}=250000, k_{D}=200
$$

The above control parameters make the system have better tracking effect on the step signal and the square wave signal.

\section{Comparison of simulation results}

Light load case: 


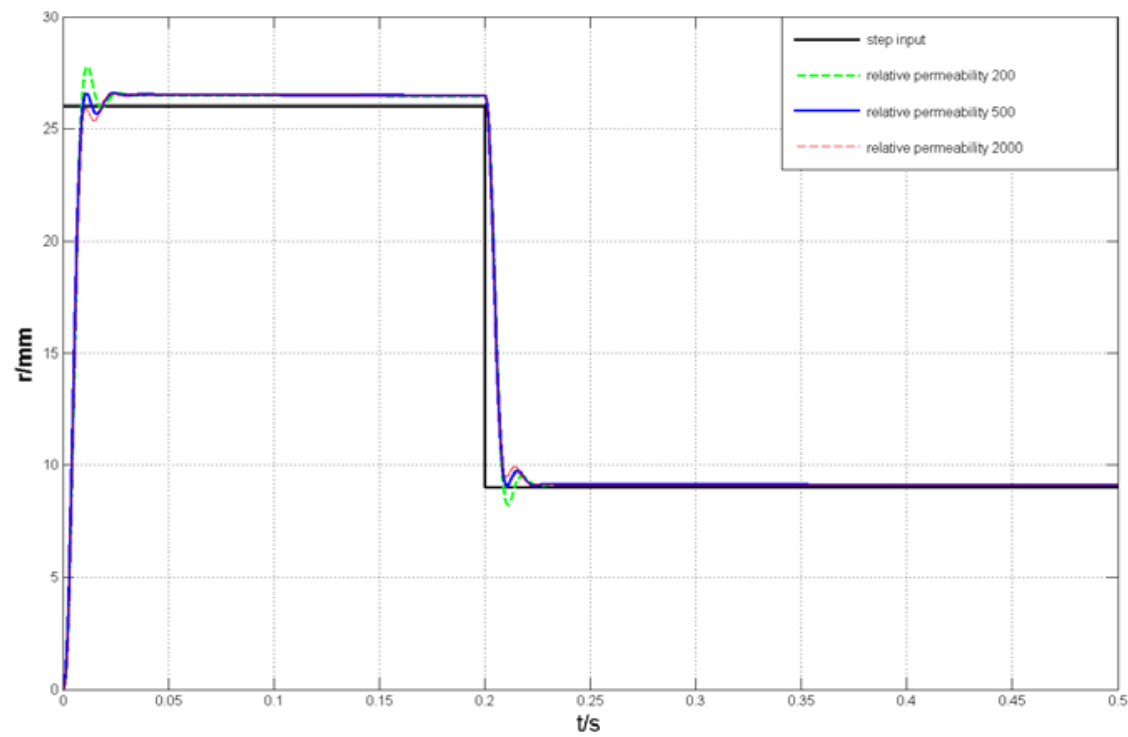

Fig 4 Step responses of different relative permeability in light load case

In light case, simulation results shows: $\mu_{r}=2000$, with the overshoot as zero and the settling time $0.022 \mathrm{~s} ; \mu_{r}=200$, the overshoot is $5 \%$ and the settling time is $0.03 \mathrm{~s}$.Comparing the simulation results of different relative permeability $\mu_{r}=2000, \mu_{r}=500$ and $\mu_{r}=200$, the truth shows that with the increase of relative permeability, the dynamic performances of suspension control system become worse.

Heavy load case:

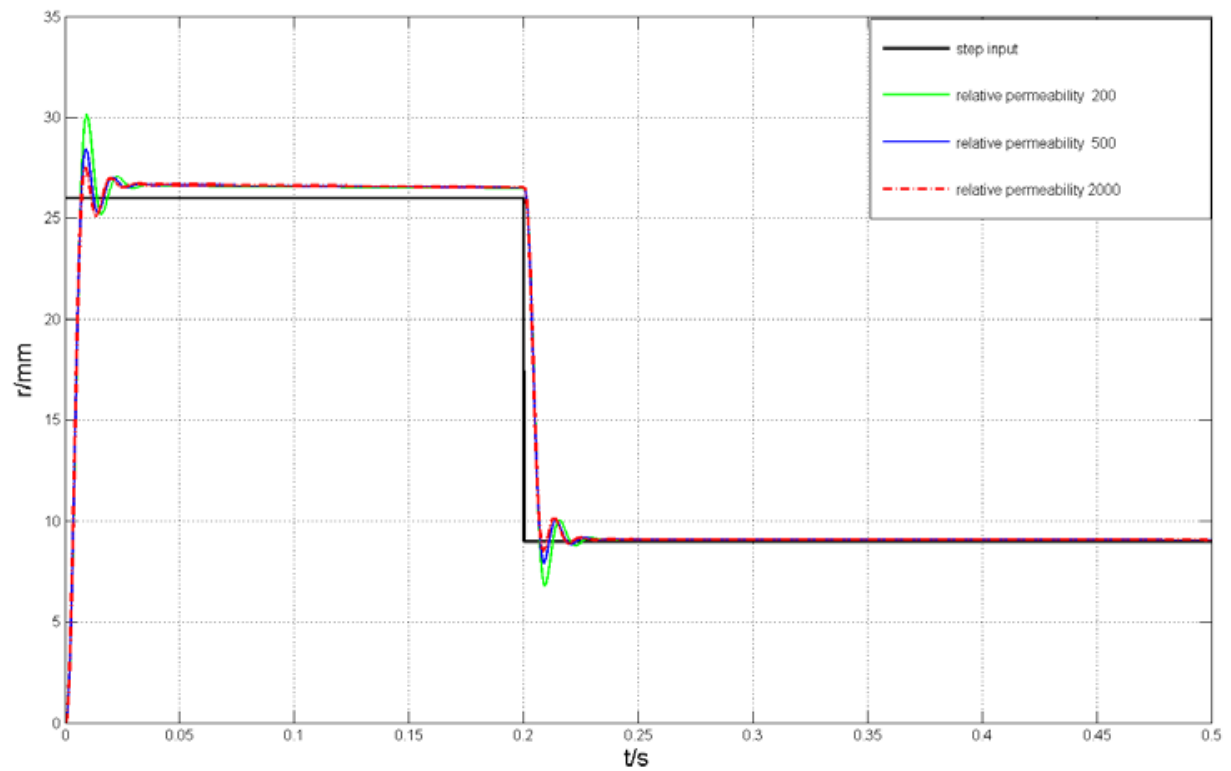

Fig 5 Step responses of different relative permeability in heavy load case

In heavy load case, simulation results shows: $\mu_{r}=2000$, with the overshoot as $5 \%$ and the settling time $0.022 \mathrm{~s} ; \mu_{r}=200$, the overshoot is $22 \%$ and the settling time is $0.028 \mathrm{~s}$. Comparing the simulation results of different relative permeability $\mu_{r}=2000 、 \mu_{r}=500$ and $\mu_{r}=200$, the truth shows that with the increase of relative permeability, the dynamic performances of suspension control system become worse.

\section{Summary}

From the results of the present study, it is available to neglect the effect of relative permeability 
on the system control performance when the relative permeability is large under both light and heavy loads. When the relative permeability is small, the stability of the control system has a certain impact. The system overshoot increases and the settling time becomes longer. In practical systems, the effect of relative permeability depends on the range of relative permeability in system working range. Therefore, it is required to select suitable track material in engineering, and to ensure that the working range of the system is in the linearity section of the track material $B-H$ curve [5].

\section{References}

[1] Long Hua She, Gui Dong Liu. Study on Dynamic Characteristics of Single Ferromagnetic Suspension Control System [J]. Electric Locomotives \& Mass Transit Vehicles, 2006, 29 (3): 17-19.

[2] Xiang Cheng Li. General magnetic permeability of ferromagnetic materials [J]. Journal of Qufu Normal University (Natural Science Edition), 1980 (3).

[3] Qiang Chen. Design of Digital Suspension Control System for Maglev Train [D]. National University of Defense Technology, 2002.

[4] Ren Ren Liang. PID parameter self-tuning algorithm in the application of suspension control system [D]. National University of Defense Technology, 2008.

[5] Lan Liu, Jin Ru Zhang. An approximate mathematical model of hysteresis loop [J]. Journal of Wuhan University of Technology, 1992 (3): 103-109. 